\title{
Quorum sensing and quorum quenching in Vibrio harveyi: lessons learned from in vivo work
}

\author{
Tom Defoirdt ${ }^{1,2}$, Nico Boon ${ }^{1}$, Patrick Sorgeloos ${ }^{2}$, Willy Verstraete ${ }^{1}$ and Peter Bossier ${ }^{2}$ \\ ${ }^{1}$ Laboratory of Microbial Ecology and Technology (LabMET), Ghent University, Ghent, Belgium and \\ ${ }^{2}$ Laboratory of Aquaculture and Artemia Reference Center, Ghent University, Ghent, Belgium
}

\begin{abstract}
Luminescent vibrios, bacteria belonging to the species Vibrio harveyi and closely related species, are important pathogens in aquaculture that can affect almost all types of cultured animals. Due to large-scale use of antibiotics, many luminescent vibrios have acquired (multiple) resistance, which render antibiotic treatments ineffective. One of the alternative strategies that has recently been developed to control infections caused by antibiotic-resistant bacteria is the disruption of quorum sensing, bacterial cell-to-cell communication. The quorum sensing system of $V$. harveyi has been studied quite intensively in vitro. Recent studies have been directed towards understanding the impact of quorum sensing and quorum sensing disruption on the virulence of luminescent vibrios towards different host organisms in vivo. This mini-review aims at discussing the current knowledge of quorum sensing in luminescent vibrios in vivo. Subsequently, quorum quenching by halogenated furanones is discussed and finally, some directions for further research are presented.
\end{abstract}

The ISME Journal (2008) 2, 19-26; doi:10.1038/ismej.2007.92; published online 25 October 2007

Subject Category: microbe-microbe and microbe-host interactions

Keywords: quorum sensing; quorum quenching; luminescent vibriosis; aquaculture

\section{Introduction}

Vibrio harveyi and closely related species such as Vibrio campbellii and Vibrio parahaemolyticus are amongst the most important bacterial pathogens in the intensive rearing of molluscs, finfish and especially shrimp (Austin and Zhang, 2006).

Vibrios are opportunists that only cause disease when the host organisms are immune-suppressed or otherwise physiologically stressed, with the frequency of infection often being attributable to adverse culture conditions (Alderman and Hastings, 1998). In our laboratories, adverse culture conditions are simulated in laboratory challenge tests by feeding the animals with a suboptimal diet (Defoirdt et al., 2005; Marques et al., 2005; Tinh et al., 2007b). In order to overcome the negative consequences of adverse culture conditions, farmers traditionally rely on the use of antibiotics (Subasinghe et al., 2001). Due to the indiscriminate misuse of antibiotics in aquaculture (Cabello, 2006), vibrios are now resistant to several antibiotics and consequently, antibiotics are no longer effective in treating

Correspondence: T Defoirdt, Laboratory of Microbial Ecology and Technology, Ghent University, Coupure Links 653, Ghent B-9000, Belgium.

E-mail: Tom.Defoirdt@UGent.be luminescent vibriosis. Karunasagar et al. (1994), for instance, reported mass mortality in tiger shrimp (Penaeus monodon) larvae caused by $V$. harveyi strains with multiple antibiotic resistance that was linked to the use of antibiotics in hatcheries. Hence, the quest for alternative methods to control infections caused by antibiotic-resistant bacteria is an important challenge for the sustainable development of aquaculture.

\section{Quorum sensing-bacterial cell-to-cell communication}

One of the new strategies that has been proposed to control infections in aquaculture is disruption of quorum sensing, bacterial cell-to-cell communication by means of small signal molecules (Defoirdt et al., 2004). Quorum sensing is a mechanism of gene regulation in which bacteria coordinate the expression of certain genes in response to the presence or absence of small signal molecules. This mechanism was first discovered in the marine bacterium Vibrio fischeri (Nealson et al., 1970) and was thought to be restricted to only a limited number of species. Later on, similar systems were found to be present in many other bacteria. 
The hypothesis that prevails in literature is that bacteria use quorum sensing to sense population density (Miller and Bassler, 2001). According to this hypothesis, quorum sensing-regulated genes are expressed (or repressed) depending on the bacterial cell density. However, this hypothesis has never been proven and is still under debate. Redfield (2002) argued for a more direct function of quorum sensing: the ability to determine whether excreted molecules rapidly diffuse away from the cell. This diffusion sensing would allow cells to regulate excretion of degradative enzymes and other gene products in such a way as to minimize losses owing to extracellular diffusion and mixing.

Quorum sensing-regulated gene expression is most often studied in vitro (that is, in bacterial cultures grown in liquid or on solid growth medium). However, microbiologists are becoming more and more aware of the fact that this gene regulation is linked to and influenced by environmental and host-derived signals (Newton and Fray, 2004). This mini-review aims at discussing the current knowledge of quorum sensing and quorum quenching in $V$. harveyi and closely related bacteria in vivo during Vibrio-animal interactions.

\section{The Quorum sensing system of $\boldsymbol{V}$. harveyi}

$V$. harveyi has been found to use a three-channel quorum sensing system (Figure 1). The first channel of this system is mediated by the harveyi autoinducer 1 (HAI-1), an acylated homoserine lactone (AHL) (Cao and Meighen, 1989). The second channel is mediated by the so-called autoinducer 2 (AI-2), which is a furanosyl borate diester (Chen et al., 2002). The chemical structure of the third autoinducer, called cholerae autoinducer 1 (CAI-1), is still unknown. The autoinducers are detected at the cell surface by membrane-bound, two-component receptor proteins that feed a common phosphorylation/dephosphorylation signal transduction cascade (Taga and Bassler, 2003). Central in the signal transduction cascade is the LuxO protein. Phosphorylated LuxO indirectly inhibits production of the transcriptional regulator protein LuxR $_{\mathrm{Vh}}$ through the action of five small regulatory RNAs (Tu and Bassler, 2007). LuxR $\mathrm{Vh}_{\mathrm{Vh}}$ directly activates the lux operon (Swartzman et al., 1992), whereas the majority of other quorum sensing-regulated genes appears to be indirectly controlled by $\operatorname{LuxR}_{\mathrm{Vh}}$ (Waters and Bassler, 2007). Tu and Bassler (2007) recently proposed that the multiple small regulatory RNAs function to translate increasing autoinducer concentrations into a precise gradient of $\mathrm{LuxR}_{\mathrm{Vh}}$, resulting in a gradient of expression of quorum sensing-regulated target genes. In other words, the concentration of LuxR ${ }_{V h}$ depends on the concentration of the five small regulatory RNAs, which is determined by the phosphorylation status of LuxO. The phosphorylation status of LuxO in its turn is determined by the net result of the kinase and phosphatase activities of the three receptors and thus dependent on the concentration of the three autoinducers.

Interestingly, Waters and Bassler (2007) recently reported that different quorum sensing-controlled a

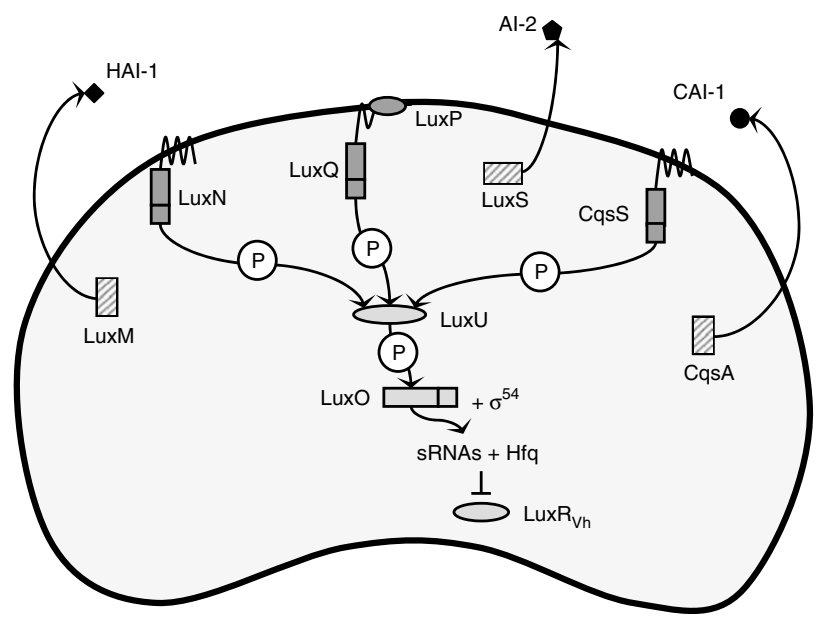

b

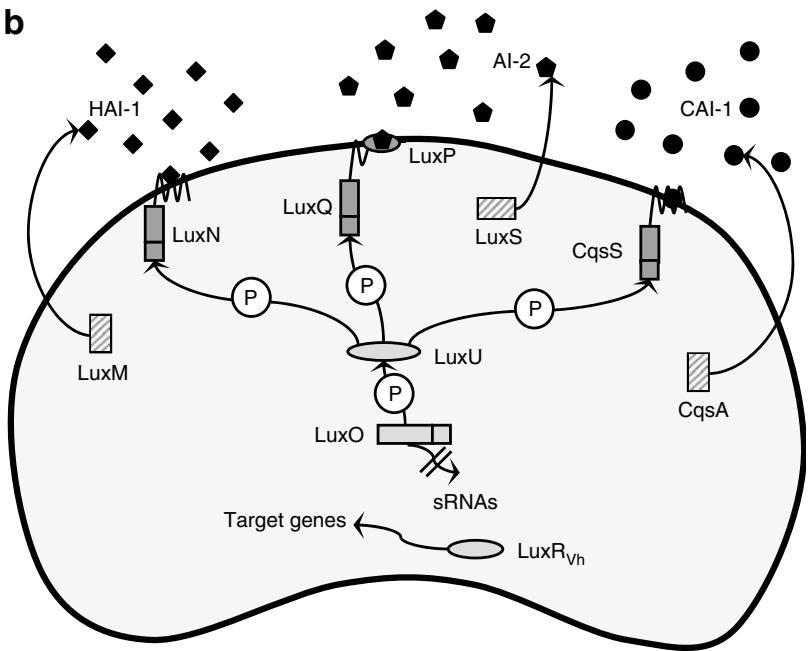

Figure 1 Quorum sensing in Vibrio harveyi. The LuxM, LuxS and CqsA enzymes synthesize the autoinducers harveyi autoinducer 1 (HAI-1), autoinducer 2 (AI-2) and cholerae autoinducer 1 (CAI-1), respectively. These autoinducers are detected at the cell surface by the LuxN, LuxQ and CqsS two-component receptor proteins, respectively. Detection of AI-2 by LuxQ requires the periplasmic protein LuxP. (a) In the absence of autoinducers, the receptors autophosphorylate and transfer phosphate to LuxO via LuxU. Phosphorylation activates LuxO, which together with $\sigma^{54}$ activates the production of five small regulatory RNAs (sRNAs). These sRNAs, together with the chaperone Hfq, destabilize the mRNA encoding the transcriptional regulator LuxR $\mathrm{Vh}_{\mathrm{Vh}}$. Therefore, in the absence of autoinducers, the LuxR $_{\mathrm{Vh}}$ protein is not produced. (b) In the presence of high concentrations of the autoinducers, the receptor proteins switch from kinases to phosphatases, which result in dephosphorylation of LuxO. Dephosphorylated LuxO is inactive and therefore, the sRNAs are not formed and the transcriptional regulator LuxR $\mathrm{Vh}_{\mathrm{Vh}}$ is produced. See text for more details. () denotes phosphotransfer. 
genes are differentially regulated by a given autoinducer input state, although they all depend on the LuxR $_{\mathrm{Vh}}$ concentration. The authors explained this by the target promoters having different affinities for LuxR $_{\mathrm{Vh}}$ and distinguished three classes of target genes. Genes that respond fully to either one of the autoinducers (resulting in relatively low $\mathrm{LuxR}_{\mathrm{Vh}}$ levels) were predicted to have promoters with high affinity for $\operatorname{LuxR}_{\mathrm{Vh}}$. Genes showing an additive response to the autoinducers (that is, significant expression in the presence of either one of the autoinducers, but a full response only in the presence of all autoinducers) were predicted to have intermediate affinity for LuxR $R_{\mathrm{Vh}}$. Genes only responding to the coincident presence of the autoinducers were suggested to have low affinity for LuxR $_{\mathrm{Vh}}$ since they require the highest concentration of $\operatorname{LuxR}_{\mathrm{Vh}}$.

\section{The impact of quorum sensing on the virulence of $V$. harveyi in vivo}

Phenotypes that were found to be controlled by the $V$. harveyi quorum sensing system in vitro include bioluminescence (Bassler et al., 1993) and the production of several virulence factors such as a type III secretion system (Henke and Bassler, 2004a), extracellular toxin (Manefield et al., 2000), metalloprotease (Mok et al., 2003) and a siderophore (Lilley and Bassler, 2000). Recently, using quorum sensing mutants, we found that the AI-2-mediated channel of the $V$. harveyi quorum sensing system regulates virulence of the bacterium towards the brine shrimp Artemia franciscana in vivo (Defoirdt et al., 2005). Indeed, inactivation of the AI-2 synthase gene luxS or the AI-2 receptor gene luxP abolished mortality in brine shrimp larvae caused by a pathogenic $V$. harveyi strain. In contrast, inactivation of the HAI-1-mediated channel of the system had no effect on virulence of the strain towards the shrimp. The effect of the CAI-1-mediated channel was not studied since CAI-1 signalling was not yet described at the moment the experiments were performed. However, in later experiments, none of the signal molecule synthase double mutants MM77 (HAI- ${ }^{-}$, AI-2 ${ }^{-}, \mathrm{CAI}_{-1}{ }^{+}$), JMH605 (HAI-1 ${ }^{-}, \mathrm{AI}-2^{+}, \mathrm{CAI}^{-1}{ }^{-}$) and JMH606 (HAI-1 ${ }^{+}$, AI- $\left.2^{-}, \mathrm{CAI}-1^{-}\right)$was found to cause mortality in brine shrimp (our unpublished results). Although it would be more illustrative to test the virulence of a single CAI-1 negative mutant, these observations suggest that disruption of the CAI-1-mediated channel probably will have the same effect as observed for the AI-2-mediated channel. It therefore seems that both AI-2 and CAI-1 are necessary for virulence towards brine shrimp, whereas HAI-1 is not.

The inactivity of the HAI-1-mediated channel of the $V$. harveyi quorum sensing system during infection of brine shrimp could be explained by the signal either not being produced or having a low stability in vivo. The first possibility would indicate that $V$. harveyi can alter the production of signals in accordance to the environment (and thus according to the type of animal during infection). Instability of HAI-1 could be either due to environmental conditions or host-derived enzyme activity. HAI-1 is an AHL, and this type of signal molecules has been shown to be unstable at relatively high $\mathrm{pH}$, with short-acyl chain AHLs (such as V. harveyi HAI-1) being the most unstable ones (Byers et al., 2002; Yates et al., 2002). Although the $\mathrm{pH}$ of the brine shrimp gut is unknown, it might be sufficiently alkaline to hydrolize the HAI-1 signal and as a consequence, it would fail to activate the HAI-1 receptor. Given the fact that different higher organisms have been reported to produce AHL-inactivating enzyme activity (Chun et al., 2004; Yang et al., 2005), an alternative explanation could be that the shrimp produces enzymes that inactivate AHL-type of quorum sensing molecules.

The $V$. harveyi quorum sensing system has been described as a three-way detector, with the expression of quorum sensing-regulated genes being proportional to the levels of the three signal molecules (Henke and Bassler, 2004b). Apparently, the detection of AI-2 and CAI-1 results in sufficiently high levels of the $\mathrm{LuxR}_{\mathrm{Vh}}$ transcriptional regulator to allow expression of the virulence factors that are essential to kill brine shrimp, whereas the LuxR $_{\mathrm{Vh}}$ concentration produced in the presence of only one of these two signal molecules is not. Interestingly, Tinh et al. (2007b) found that both HAI-1 and AI-2 signalling needed to be inactivated in order to neutralize the negative effects of $V$. harveyi BB120 towards gnotobiotic rotifers (Brachionus plicatilis). This indicates that, in contrast to what was found in brine shrimp, HAI-1 signalling is active in the rotifers and suggests that higher levels of the $\operatorname{LuxR}_{\mathrm{Vh}}$ transcriptional regulator are needed to infect these organisms when compared to brine shrimp. Alternatively, although all currently known quorum sensing-regulated genes are controlled by the model as described in Figure 1, we cannot exclude the possibility that pathogenicity to rotifers is mediated by a specific virulence factor that is regulated by HAI-1 through a yet unknown signal transduction loop.

\section{On the value of a three-channel quorum sensing system}

The above-mentioned in vivo work seems to confirm the hypothesis of Taga and Bassler (2003) that $V$. harveyi uses a three-channel quorum sensing system in order to counteract bias. Indeed, if quorum sensing-regulated gene expression would be based on the detection of only one signal molecule, then bias could be caused by inactivation of the signal, either chemically (for example, AHLs such as HAI-1 are unstable at high $\mathrm{pH}$; Byers et al. (2002)) or 
biologically (for example, HAI-1 could be degraded by Bacillus spp.; Dong et al. (2002)). Indeed, the HAI-1-mediated channel of the $V$. harveyi quorum sensing system seems to be inactive during infection of brine shrimp (see above). Consequently, the vibrios would fail to infect brine shrimp if they would only rely on HAI-1 signalling. On the other hand, interference due to signal production by bacteria belonging to other species (for example, in case of AI-2) will not drastically bias the threechannel system since the two other signals are needed as well for maximal activation (Henke and Bassler, 2004b). Moreover, this kind of bias would not be so dramatic since activation of the quorum sensing cascade by a signal produced by other bacteria would indicate that mixing and diffusion in the micro-environment is low and hence, the vibrios would have a high chance to benefit from their own quorum sensing-activated gene products (according to the diffusion sensing hypothesis of Redfield (2002)).

\section{Quorum sensing disruption by halogenated furanones}

Halogenated furanones have been shown before to disrupt AHL- as well as AI-2-mediated signalling in Gram-negative bacteria, without affecting growth (Ren et al., 2001; Hentzer and Givskov, 2003). Consistent with this, the natural furanone (5Z)-4bromo-5-(bromomethylene)-3-butyl-2(5H)-furanone, added to the culture water at $20 \mathrm{mg} \mathrm{l}^{-1}$, was shown to increase the survival of brine shrimp larvae challenged to different pathogenic isolates belonging to the species $V$. campbellii, $V$. harveyi and V. parahaemolyticus (Defoirdt et al., 2006). This suggests that virulence attenuation caused by quorum sensing disruption is a general feature for luminescent vibrios and not specific for one strain. For some of the strains, the protection offered by the furanone treatment was complete, whereas other strains still caused significant mortality in furanonetreated larvae. Hence, apparently there is a difference between different strains in the degree of quorum sensing disruption by the compound. Unfortunately, it was not possible to investigate whether higher furanone concentrations could protect the larvae from the isolates that still caused mortality at $20 \mathrm{mgl}^{-1}$ of furanone because the compound was highly toxic to the larvae at $50 \mathrm{mg} \mathrm{l}^{-1}$ (Defoirdt et al., 2006). This implies that the therapeutic index for the furanone is too low to be applied in practice. Hentzer and Givskov (2003) also mentioned that the currently known halogenated furanones are too reactive for the treatment of infections in higher organisms.

Initial in vitro bioluminescence experiments showed that halogenated furanones block HAI-1and AI-2-mediated signalling in $V$. harveyi (Ren et al., 2001; Defoirdt et al., 2006). It was originally hypothesized that the compounds disrupt quorum sensing in this bacterium by displacing the signal molecules from their receptors (Manefield et al., 2000; Ren et al., 2001). However, recent research at our laboratories showed that the natural furanone (5Z)-4-bromo-5-(bromomethylene)-3-butyl-2(5H)-

furanone blocks quorum sensing-regulated gene expression in $V$. harveyi by decreasing the DNAbinding activity of the quorum sensing transcriptional regulator $\operatorname{LuxR}_{\mathrm{Vh}}$ and not by interacting with the signal molecule receptors (Defoirdt et al., 2007). The fact that the furanone affects the master regulator rather than selectively blocking one of the channels of the $V$. harveyi quorum sensing system is quite important with respect to possible practical applications. As mentioned earlier, there seems to be a difference in the relative importance of the three channels for a successful infection of different hosts. Hence, since the furanone blocks all three channels of the system at once by acting at the end of the quorum sensing signal transduction cascade, it will not be necessary to develop different furanone compounds to protect different hosts. Indeed, the natural furanone (5Z)-4-bromo-5-(bromomethylene)-3-butyl-2(5H)-furanone has been shown to protect both brine shrimp and rotifers against luminescent vibrios (Defoirdt et al., 2006; Tinh et al., 2007b).

Interestingly, the natural furanone (5Z)-4-bromo5-(bromomethylene)-3-butyl-2(5H)-furanone had no effect on growth of the vibrios (Manefield et al., 2000; Defoirdt et al., 2006), indicating that it poses no (or very small) selective pressure on the bacteria. Consequently, the chance of resistance development will probably be smaller than for conventional antibiotics. Because quorum sensing disrupting compounds attenuate virulence of pathogenic bacteria without affecting growth, they have been termed antipathogenic drugs, as opposed to antibacterial drugs (Hentzer and Givskov, 2003). Antipathogenic drugs target key regulatory systems in bacterial pathogens that regulate the expression of virulence factors. The fact that antipathogenic compounds are unlikely to pose a selective pressure for the development of resistance, makes this concept highly attractive as a sustainable biocontrol strategy. Hence, it is certainly worthwhile to try synthesizing analogous compounds with lower toxicity, which could then be applied in practice.

\section{Directions for further research}

Biodegradation of signal molecules

AI-2 is produced from $S$-adenosylmethionine (SAM) in three enzymatic steps (Figure 2). When SAM is used as a methyl donor, $S$-adenosylhomocysteine (SAH) is produced. The enzyme Pfs converts SAH to $S$-ribosylhomocysteine (SRH), and subsequently, LuxS acts on SRH to make homocysteine and 4,5-dihydroxy-2,3-pentanedione (DPD) (Schauder et al., 2001). 
a SAM

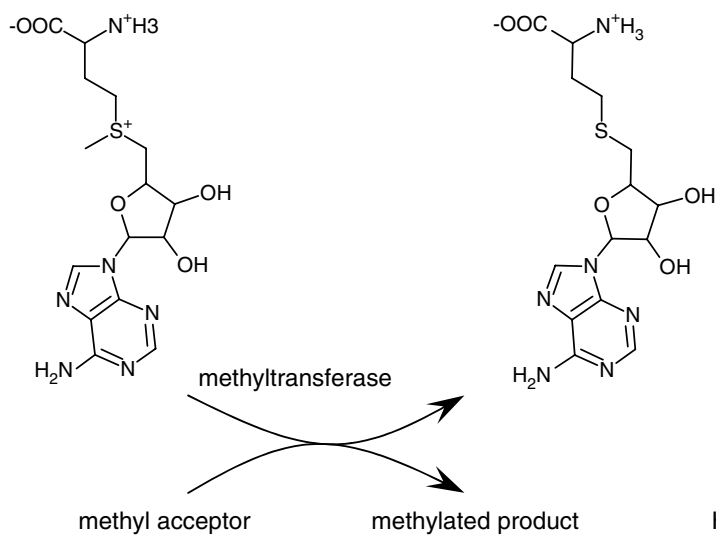

SAH

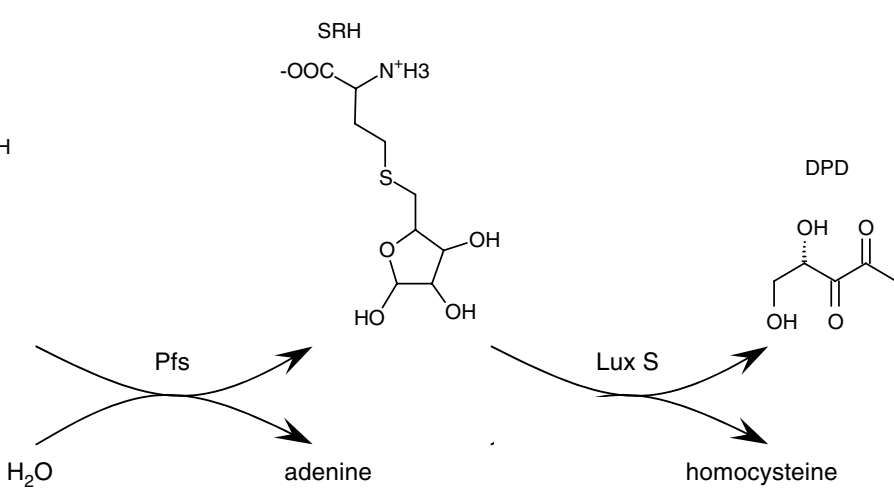

b

b

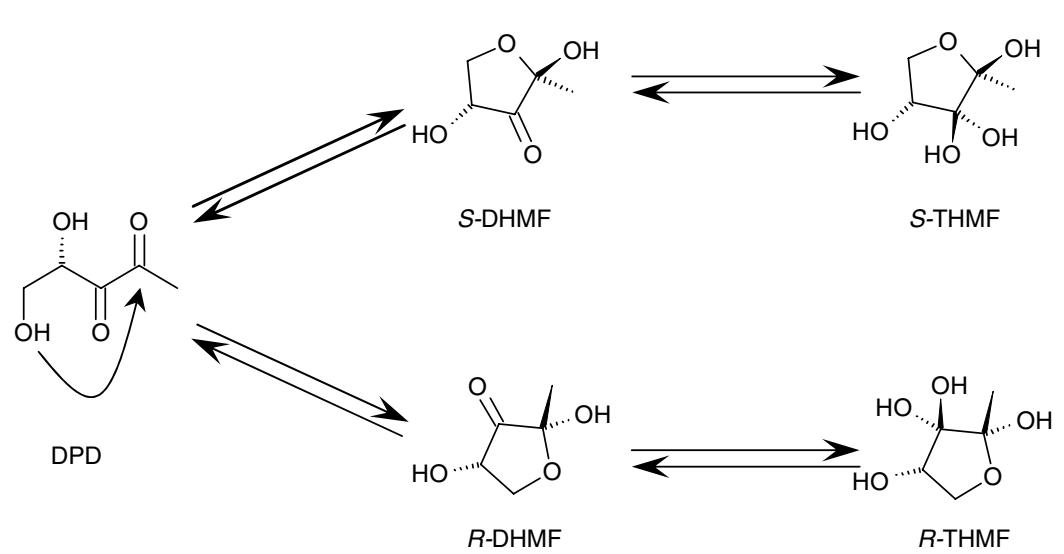

Figure 2 Biosynthesis of AI-2. (a) 4,5-dihydroxy-2,3-pentanedione (DPD), the precursor to all AI-2, is synthesized from $S$ adenosylmethionine (SAM) in three enzymatic steps. SAH, $S$-adenosylhomocysteine; SRH, S-ribosylhomocysteine. (b) DPD rearranges and undergoes further reactions (all equilibria) to form distinct biologically active signal molecules that are generically termed AI-2. Vibrio harveyi AI-2 is produced by the upper pathway; Salmonella typhimurium AI-2 by the lower one. $S$-DHMF: $(2 S, 4 S)$-dihydroxy-2methyldihydro-3-furanone; $R$-DHMF, $(2 R, 4 S)$-dihydroxy-2methyldihydro-3-furanone; $S$-THMF, $(2 S, 4 S)$-2-methyl-2,3,3,4-tetra-hydroxytetrahydrofuran; $R$-THMF, (2R,4S)-2-methyl-2,3,3,4-tetrahydroxytetrahydro-furan (based on Vendeville et al. (2005); De Keersmaecker et al. (2006)).

In $V$. harveyi, DPD cyclizes, is hydrated and is converted into the active AI-2 signal molecule (Chen et al., 2002).

More recent research showed that the active AI-2 signal in Salmonella typhimurium has a different chemical structure when compared to $V$. harveyi AI-2 (Miller et al., 2004). In S. typhimurium, AI-2 induces transcription of the IsrACDBFGE operon. The first four genes in this operon encode an ABC transporter, through which AI-2 is imported into the cells (Taga et al., 2003). The other genes of the operon, together with the lsrK en lsrR genes, encode proteins that phosphorylate AI-2 and further degrade the signal (Taga and Bassler, 2003). A similar system has been described in Escherichia coli (Xavier and Bassler, 2005b). It is still unclear why these two species produce a signal, which then activates its own degradation. The signal might be used as a nutrient, although the bacteria cannot grow in minimal media containing $\mathrm{AI}-2$ as the sole carbon source (Taga and Bassler, 2003). Another hypothesis is that by degrading AI-2, these bacteria trick their competitors into behaving as if there were no AI-2 (Federle and Bassler, 2003). In an exciting report, Xavier and Bassler (2005a) studied AI-2 cross talk between $V$. harveyi and $E$. coli and found that when co-cultured, $V$. harveyi produced only $18 \%$ of the bioluminescence it produced in pure culture. The effect was shown to be due to internalization and degradation of AI-2 by E. coli since no reduction occurred in co-cultures with mutants that are defective in AI-2 internalization.

The degradation of AI-2 by E. coli is not constitutive but is under control of several regulatory mechanisms, such as cAMP-CRP, the repressor LsrR and RpoS (De Keersmaecker et al., 2006), which makes it inappropriate for practical applications. Since the different active AI-2 signal molecules are all in equilibrium with each other and with DPD (see Figure 2), inactivation of one of them will result in a decrease in the concentrations of all the other forms. However, no other bacteria than E. coli and 
S. typhimurium have been reported that are able to degrade AI-2 or its precursor DPD. The enrichment, isolation and identification of AI-2- or DPD-degrading bacteria constitutes an intriguing area for further research.

Several reports have been published describing the degradation of AHLs by different bacteria. Enzymes that are able to inactivate AHLs have been discovered in different Gram-negative species belonging to the $\alpha$-proteobacteria (Zhang et al., 2002), the $\beta$-proteobacteria (Leadbetter and Greenberg, 2000; Lin et al., 2003; Uroz et al., 2003) and the $\gamma$ proteobacteria (Uroz et al., 2003) as well as in some Gram-positive species (Dong et al., 2002; Lee et al., 2002; Uroz et al., 2003). Interestingly, Tinh et al. (2007a) very recently isolated AHL-degrading enrichment cultures from the digestive tract of healthy Pacific white shrimp (Penaeus vannamei). The enrichment cultures protected rotifers from the negative effects of an AI-2 non-producing $V$. harveyi mutant, suggesting that the bacteria interfered with HAI-1-regulated gene expression in vivo. Also Bacillus strains that are currently used as probiotics in aquaculture (for example, Moriarty, 1998) would be worthwhile testing in this respect since different Bacillus spp. have been reported to degrade AHLs (Dong et al., 2002; Lee et al., 2002). It would be highly interesting to further investigate whether signal molecule-degrading bacteria (either AI-2 degraders alone or both AHL and AI-2 degraders together, depending on the host system) would be capable to disrupt $V$. harveyi quorum sensing and whether this could protect cultured animals from luminescent vibriosis. If they would be effective in controlling luminescent vibriosis, these bacteria could be used as a new kind of probiotics.

\section{Inhibition of signal molecule biosynthesis}

Another possibility to disrupt quorum sensing is the inhibition of signal molecule biosynthesis. This strategy has already been tested in the AHLmediated quorum sensing system of Pseudomonas aeruginosa by applying substrate analogues for the AHL synthase enzyme RhlI (Parsek et al., 1999). Interestingly, Alfaro et al. (2004) synthesized two inhibitors of the AI-2 synthase enzyme LuxS, $S$ anhydroribosyl-L-homocysteine and $S$-homoribosylL-cysteine. Both compounds are analogues of the LuxS substrate $S$-ribosyl-L-homocysteine. The compounds were tested in vitro using purified LuxS and were found to inhibit AI-2 production by the enzyme. Very recently, Shen et al. (2006) synthesized the first LuxS inhibitors with activity in the submicromolar range, with $\mathrm{K}_{\mathrm{I}}$ values as low as $0.43 \mu \mathrm{M}\left(\approx 115 \mu \mathrm{g} \mathrm{l}^{-1}\right)$. It would be interesting to test whether signal molecule synthase inhibitors could disrupt quorum sensing in $V$. harveyi and if they do, whether they have any impact on the virulence of luminescent vibrios towards aquatic organisms.

\section{Acknowledgements}

We thank the 'Instituut voor de aanmoediging van Innovatie door Wetenschap en Technologie in Vlaanderen' (IWT-Vlaanderen) and the 'Fonds voor Wetenschappelijk Onderzoek' (TD is a post-doctoral fellow of FWO-Vlaanderen) for financial support.

\section{References}

Alderman DJ, Hastings TS. (1998). Antibiotic use in aquaculture: development of antibiotic resistancepotential for consumer health risks. Int J Food Sci Technol 33: 139-155.

Alfaro JF, Zhang T, Wynn DP, Karschner EL, Zhou ZS. (2004). Synthesis of LuxS inhibitors targeting bacterial cell-cell communication. Org Lett 6: 3043-3046.

Austin B, Zhang XH. (2006). Vibrio harveyi: a significant pathogen of marine vertebrates and invertebrates. Lett Appl Microbiol 43: 119-124.

Bassler BL, Wright M, Showalter RE, Silverman MR. (1993). Intercellular signaling in Vibrio harveyisequence and function of genes regulating expression of luminescence. Mol Microbiol 9: 773-786.

Byers JT, Lucas C, Salmond GPC, Welch M. (2002). Nonenzymatic turnover of an Erwinia carotovora quorum-sensing signaling molecule. J Bacteriol 184: 1163-1171.

Cabello FC. (2006). Heavy use of prophylactic antibiotics in aquaculture: a growing problem for human and animal health and for the environment. Environ Microbiol 8: 1137-1144.

Cao JG, Meighen EA. (1989). Purification and structural identification of an autoinducer for the luminescence system of Vibrio harveyi. J Biol Chem 264: 2167021676.

Chen X, Schauder S, Potier N, Van Dorsselaer A, Pelczer I, Bassler BL et al. (2002). Structural identification of a bacterial quorum-sensing signal containing boron. Nature 415: 545-549.

Chun CK, Ozer EA, Welsh MJ, Zabner J, Greenberg EP. (2004). Inactivation of a Pseudomonas aeruginosa quorum-sensing signal by human airway epithelia. Proc Natl Acad Sci USA 101: 3587-3590.

Defoirdt T, Boon N, Bossier P, Verstraete W. (2004). Disruption of bacterial quorum sensing: an unexplored strategy to fight infections in aquaculture. Aquaculture 240: 69-88.

Defoirdt T, Bossier P, Sorgeloos P, Verstraete W. (2005). The impact of mutations in the quorum sensing systems of Aeromonas hydrophila, Vibrio anguillarum and Vibrio harveyi on their virulence towards gnotobiotically cultured Artemia franciscana. Environ Microbiol 7: 1239-1247.

Defoirdt T, Crab R, Wood TK, Sorgeloos P, Verstraete W, Bossier P. (2006). Quorum sensing-disrupting brominated furanones protect the gnotobiotic brine shrimp Artemia franciscana from pathogenic Vibrio harveyi, Vibrio campbellii and Vibrio parahaemolyticus isolates. Appl Environ Microbiol 72: 6419-6423.

Defoirdt T, Miyamoto C, Wood TK, Meighen EA, Sorgeloos $\mathrm{P}$, Verstraete $\mathrm{W}$ et al. (2007). The natural furanone (5Z)4-bromo-5-(bromomethylene)-3-butyl-2( $5 H$ )-furanone disrupts quorum sensing-regulated gene expression in Vibrio harveyi by decreasing the DNA-binding activity 
of the transcriptional regulator protein luxR. Environ Microbiol 9: 2486-2495.

De Keersmaecker SCJ, Sonck K, Vanderleyden J. (2006). Let LuxS speak up in AI-2 signaling. Trends Microbiol 14: 114-119.

Dong YH, Gusti AR, Zhang Q, Xu JL, Zhang LH. (2002). Identification of quorum-quenching $N$-acyl homoserine lactonases from Bacillus species. Appl Environ Microbiol 68: 1754-1759.

Federle MJ, Bassler BL. (2003). Interspecies communication in bacteria. I Clin Invest 112: 1291-1299.

Henke JM, Bassler BL. (2004a). Quorum sensing regulates type III secretion in Vibrio harveyi and Vibrio parahaemolyticus. J Bacteriol 186: 3794-3805.

Henke JM, Bassler BL. (2004b). Three parallel quorum sensing systems regulate gene expression in Vibrio harveyi. J Bacteriol 186: 6902-6914.

Hentzer M, Givskov M. (2003). Pharmacological inhibition of quorum sensing for the treatment of chronic bacterial infections. J Clin Invest 112: 1300-1307.

Karunasagar I, Pai R, Malahti GR, Karunasagar I. (1994). Mass mortality of Penaeus monodon larvae due to antibiotic-resistant Vibrio harveyi infection. Aquaculture 128: 203-209.

Leadbetter JR, Greenberg EP. (2000). Metabolism of acyl-homoserine lactone quorum-sensing signals by Variovorax paradoxus. J Bacteriol 182: 6921-6926.

Lee SJ, Park SY, Lee JJ, Yum D, Koo BT, Lee JK. (2002). Genes encoding the $N$-acyl homoserine lactone-degrading enzyme are widespread in many subspecies of Bacillus thuringiensis. Appl Environ Microbiol 68: 3919-3924.

Lilley BN, Bassler BL. (2000). Regulation of quorum sensing in Vibrio harveyi by LuxO and $\sigma 54 . \mathrm{Mol}$ Microbiol 36: 940-954.

Lin YH, Xu JL, Hu J, Wang LH, Ong SL, Leadbetter JR et al. (2003). Acyl-homoserine lactone acylase from Ralstonia sp. strain XJ12B represents a novel and potent class of quorum-quenching enzymes. Mol Microbiol 47: 849-860.

Manefield M, Harris L, Rice SA, de Nys R, Kjelleberg S. (2000). Inhibition of luminescence and virulence in the black tiger prawn (Penaeus monodon) pathogen Vibrio harveyi by intercellular signal antagonists. Appl Environ Microbiol 66: 2079-2084.

Marques A, Dinh T, Ioakeimidis C, Huys G, Swings J, Verstraete W et al. (2005). Effects of bacteria on Artemia franciscana cultured in different gnotobiotic environments. Appl Environ Microbiol 71: 4307-4317.

Miller MB, Bassler BL. (2001). Quorum sensing in bacteria. Annu Rev Microbiol 55: 165-199.

Miller ST, Xavier KB, Campagna SR, Taga ME, Semmelhack MF, Bassler BL et al. (2004). Salmonella typhimurium recognizes a chemically distinct form of the bacterial quorum-sensing signal AI-2. Mol Cell 15: $677-687$.

Mok KC, Wingreen NS, Bassler BL. (2003). Vibrio harveyi quorum sensing: a coincidence detector for two autoinducers controls gene expression. EMBO $J \mathbf{2 2}$ : 870-881.

Moriarty DJW. (1998). Control of luminous Vibrio species in penaeid aquaculture ponds. Aquaculture 164: 351-358.

Nealson KH, Platt T, Hastings JW. (1970). Cellular control of the synthesis and activity of the bacterial luminescence system. J Bacteriol 104: 313-322.
Newton JA, Fray RG. (2004). Integration of environmental and host-derived signals with quorum sensing during plant-microbe interactions. Cell Microbiol 6: 213-224.

Parsek MR, Val DL, Hanzelka BL, Cronan Jr JR, Greenberg EP. (1999). Acyl homoserine-lactone quorum-sensing signal generation. Proc Natl Acad Sci USA 96: 43604365.

Redfield RJ. (2002). Is quorum sensing a side effect of diffusion sensing? Trends Microbiol 10: 365-370.

Ren D, Sims J, Wood TK. (2001). Inhibition of biofilm formation and swarming of Escherichia coli by (5Z)-4bromo-5-(bromomethylene)-3-butyl-2(5H)-furanone. Environ Microbiol 3: 731-736.

Schauder S, Shokat K, Surette MG, Bassler BL. (2001). The LuxS family of bacterial autoinducers: biosynthesis of a novel quorum-sensing signal molecule. Mol Microbiol 41: 463-476.

Shen G, Rajan R, Zhu J, Bell CE, Pei D. (2006). Design and synthesis of substrate and intermediate analogue inhibitors of $S$-ribosylhomocysteinase. J Med Chem 49: 3003-3011.

Subasinghe RP, Bondad-Reantaso MG, McGladdery SE. (2001). Aquaculture development, health and wealth. In: Subasinghe RP, Bueno P, Phillips MJ, Hough C, McGladdery SE, Arthur JR (eds). Aquaculture in the Third Millennium. Technical Proceedings of the Conference on Aquaculture in the Third Millennium. NACA Bangkok and FAO: Rome, pp 167-191.

Swartzman E, Silverman M, Meighen EA. (1992). The luxR gene product of Vibrio harveyi is a transcriptional activator of the lux promoter. J Bacteriol 174 7490-7493.

Taga ME, Bassler BL. (2003). Chemical communication among bacteria. Proc Natl Acad Sci USA 100: 1454914554.

Taga ME, Miller ST, Bassler BL. (2003). Lsr-mediated transport and processing of AI-2 in Salmonella typhimurium. Mol Microbiol 50: 1411-1427.

Tinh NTN, Gunasekara RAYSA, Boon N, Dierckens K, Sorgeloos P, Bossier P. (2007a). N-acyl homoserine lactone-degrading microbial enrichment cultures isolated from Penaeus vannamei shrimp gut and their probiotic properties in Brachionus plicatilis cultures. FEMS Microbiol Ecol 62: 45-53.

Tinh NTN, Linh ND, Wood TK, Dierckens K, Sorgeloos P, Bossier P. (2007b). Interference with the quorum sensing systems in a Vibrio harveyi strain alters the growth rate of gnotobiotically cultured rotifer Brachionus plicatilis. J Appl Microbiol 103: 194-203.

Tu KC, Bassler BL. (2007). Multiple small RNAs act additively to integrate sensory information and control quorum sensing in Vibrio harveyi. Genes Dev 21: 221-233.

Uroz S, D’ Angelo-Picard C, Carlier A, Elasri M, Sicot C, Petit A et al. (2003). Novel bacteria degrading $N$ acylhomoserine lactones and their use as quenchers of quorum-sensing-regulated functions of plant-pathogenic bacteria. Microbiology 149: 1981-1989.

Vendeville A, Winzer K, Haurlier K, Tang CM, Hardie KR. (2005). Making 'sense' of metabolism: autoinducer-2, LuxS and pathogenic bacteria. Nat Rev Microbiol 3: 383-396.

Waters CM, Bassler BL. (2007). The Vibrio harveyi quorum-sensing system uses shared regulatory components to discriminate between multiple autoinducers. Genes Dev 20: 2754-2767. 
Xavier KB, Bassler BL. (2005a). Interference with AI-2mediated bacterial cell-cell communication. Nature 437: 750-753.

Xavier KB, Bassler BL. (2005b). Regulation of uptake and processing of the quorum-sensing autoinducer AI-2 in Escherichia coli. J Bacteriol 187: 238-248.

Yang F, Wang LH, Wang J, Dong YH, Hu JY, Zhang LH. (2005). Quorum quenching enzyme activity is widely conserved in the sera of mammalian species. FEBS Lett 579: 3713-3717.
Yates EA, Philipp B, Buckley C, Atkinson S, Chhabra SR, Sockett RE et al. (2002). $N$-acylhomoserine lactones undergo lactonolysis in a $\mathrm{pH}$-, temperature-, and acyl chain length-dependent manner during growth of Yersinia pseudotuberculosis and Pseudomonas aeruginosa. Infect Immun 70: 5635-5646.

Zhang HB, Wang LH, Zhang LH. (2002). Genetic control of quorum-sensing signal turnover in Agrobacterium tumefaciens. Proc Natl Acad Sci USA 99: 4638-4643. 